\title{
Relationship between polarization-averaged molecular-frame photoelectron angular distributions and geometry
}

\author{
Etienne Plésiat, ${ }^{1}$ Piero Decleva, ${ }^{2}$ and Fernando Martín ${ }^{1,3, *}$ \\ ${ }^{1}$ Departamento de Química, Módulo 13, Universidad Autónoma de Madrid, 28049 Madrid, Spain \\ ${ }^{2}$ Dipartimento di Scienze Chimiche, Universita' di Trieste, 34127 Trieste, and CNR-IOM, Trieste, Italy \\ ${ }^{3}$ Instituto Madrileño de Estudios Avanzados en Nanociencia (IMDEA-Nanociencia), Cantoblanco, 28049 Madrid, Spain
}

(Received 25 June 2013; published 17 December 2013)

\begin{abstract}
We present a theoretical study of vibrationally resolved and unresolved molecular-frame photoelectron angular distributions (MFPADs) resulting from $K$-shell photoionization of $\mathrm{N}_{2}, \mathrm{CO}, \mathrm{C}_{2} \mathrm{H}_{2}, \mathrm{NH}_{3}, \mathrm{CH}_{4}, \mathrm{CF}_{4}, \mathrm{BF}_{3}$, and $\mathrm{SF}_{6}$ in the range of photoelectron energies $0-500 \mathrm{eV}$. We show that the MFPADs of $\mathrm{NH}_{3}$ and $\mathrm{CH}_{4}$, averaged over the polarization direction, image the molecular geometry at very low energies but also at selected higher energies. For all other molecules, the MFPADs do not image the system's geometry. However, for molecules containing heavy atoms in the periphery, $\mathrm{CF}_{4}, \mathrm{BF}_{3}$, and $\mathrm{SF}_{6}$, and for $\mathrm{N}_{2}$ and $\mathrm{CO}$, the polarization-averaged MFPADs reflect the partial accumulation of the photoelectron density in the region surrounded by the peripheral atoms. For energies at which this accumulation occurs, the MFPADs encode information about the three dimensional arrangement of the system. In general, the polarization averaged MFPADs remain quite anisotropic even at photoelectron energies as high as $500 \mathrm{eV}$.
\end{abstract}

DOI: 10.1103/PhysRevA.88.063409

PACS number(s): 33.80.Eh, 34.10.+x, 34.50.-s

\section{INTRODUCTION}

Photoelectrons emerging from localized inner shells of molecules in gas phase are scattered coherently (diffracted) by the molecular potential, thus carrying valuable information about the structure of the isolated molecule. How to extract this information from experimental observables is a problem that has received considerable attention in the past few years, partly because methods designed to provide a temporal picture of structural changes in the gas phase, e.g., ultrafast electron diffraction [1], x-ray diffraction [2], or high-harmonic generation [3], are based on this phenomenon. Experimental techniques that make use of synchrotron radiation have also been revealed as very helpful. For example, recent work has shown that intramolecular electron diffraction arising from inner-shell photoionization of diatomic and polyatomic molecules leads to pronounced oscillations in the ratios of vibrationally resolved cross sections, from which the bond lengths of the molecule and its corresponding cation can be determined with reasonable accuracy [4-7]. The method is particularly well suited at high photoelectron energies, where the details of the molecular potential can be ignored, and therefore simple semianalytical models can be used to fit the spectra and extract structure parameters.

More sophisticated approaches aim at measuring molecular-frame photoelectron angular distributions (MFPADs) [8,9]. In these measurements, the molecule has a well-defined orientation with respect to the polarization of the light field, orientation that can be achieved by laser alignment $[10,11]$ or detection of photoelectrons in coincidence with positively charged fragments [12]. In general, MFPADs reflect those details of the molecular potential that are hidden in measurements performed in the laboratory frame (in which all information related to molecular orientation is lost). Paradoxically, the richer

\footnotetext{
*fernando.martin@uam.es
}

information contained in the MFPADs makes also their interpretation rather difficult. Thus, although simplified models have been developed to accurately extract structural information [13], the latter is often a very challenging task.

Very recently, MFPADs for the $\mathrm{CH}_{4}$ molecule have been determined by detecting the photoelectron in coincidence with the positively charged fragments that result from dissociation following prompt Auger decay of $\mathrm{CH}_{4}^{+}[14,15]$. The surprising result is that, at very low photoelectron energies, the electron is mainly ejected along the bond directions. Consequently, when the MFPAD is averaged over all polarization directions, the resulting distribution provides a direct imaging of the molecular geometry. This prediction is in agreement with results of elaborate theoretical calculations for $\mathrm{CH}_{4}, \mathrm{H}_{2} \mathrm{O}$, and $\mathrm{NH}_{3}$ within the fixed-nuclei approximation [16]. The method consisting of measuring polarization-averaged MFPADs (hereafter called PAMFPADs) at low energy is very appealing because it avoids any further processing of the experimental data, so it is worth investigating its generality by considering molecules with different atomic centers and different symmetries. It also raises the question about the range of photoelectron energies in which direct imaging is expected to be observed. The measurements of Ref. [15] show that, for $\mathrm{CH}_{4}$, the PAMFPADs become almost isotropic and hence do not image the molecular geometry at energies as low as $15 \mathrm{eV}$, but it remains to be seen if imaging is possible at higher energies and, if not, if there is still structural information that can be obtained from them. Furthermore, as current experimental techniques are able to provide vibrationally resolved MFPADs [17], it is also important to investigate if the latter can also image the molecular geometry.

In this paper, we present a systematic theoretical study of vibrationally resolved and unresolved MFPADs in $K$-shell photoionization of $\mathrm{N}_{2}, \mathrm{CO}, \mathrm{C}_{2} \mathrm{H}_{2}, \mathrm{NH}_{3}, \mathrm{CH}_{4}, \mathrm{CF}_{4}, \mathrm{BF}_{3}$, and $\mathrm{SF}_{6}$ in the range of photoelectron energies $0-500 \mathrm{eV}$. We show that the PAMFPADs of $\mathrm{NH}_{3}$ and $\mathrm{CH}_{4}$ do image the molecular geometry at very low energies but also at selected high energies. For all other molecules, the PAMFPADs do 
not provide a direct imaging of the system's geometry at almost any energy. However, for the molecules containing the heavier atomic species $\mathrm{CF}_{4}, \mathrm{BF}_{3}$, and $\mathrm{SF}_{6}$, but also $\mathrm{N}_{2}$ and $\mathrm{CO}$, and for selected energy intervals, the PAMFPADs reflect partial accumulation of the photoelectron density in the region surrounded by the peripheral atoms, which is thus related to the three-dimensional arrangement of the system.

\section{THEORETICAL METHOD}

The theoretical method has been explained in detail in previous work [7,18]. Briefly, vibrationally resolved photoionization cross sections were evaluated within the dipole and Born-Oppenheimer approximations. Bound and continuum electronic wave functions were calculated by using the staticexchange density functional theory (DFT) as described in, e.g., Refs. $[19,20]$. The electronic Kohn-Sham equations were solved by using a representation in a basis of $B$-spline functions [21]. In order to include the nuclear motion, the electronic wave functions and the corresponding potential energy surfaces were evaluated over a wide range of nuclear positions. As photoionization mainly leads to molecular cations with excitation in the symmetric stretching mode, calculations were restricted to this normal coordinate by keeping all the other normal coordinates frozen at their values of the equilibrium geometry. The vibrational wave functions were obtained by solving the corresponding vibrational Schrödinger equation in a basis of $B$ splines.

\section{RESULTS AND DISCUSSION}

Figure 1 shows the calculated total photoionization cross sections. They are in good agreement with the experimental measurements [22-28]. Except for $\mathrm{SF}_{6}$, the cross-sectional values are rather similar for all systems in a wide range of photoelectron energies, $\epsilon>70 \mathrm{eV}$. At low $\epsilon$, several structures are apparent. Those appearing just above the ionization threshold are usually assigned to shape resonances, ${ }^{1}$ while the others, which are superimposed on the rapidly decreasing background, are due to diffraction of the photoelectron by the peripheral atomic centers [18]. Figure 2 shows a comparison between calculated and experimental MFPADs of $\mathrm{CH}_{4}$ for three typical orientations of the molecule with respect to the polarization axis and for the average over polarization direction. The agreement between theoretical and experimental results $[14,15]$ is good. Our results are also in excellent agreement with the theoretical ones reported in Ref. [16], which were obtained in the fixed-nuclei approximation. From this comparison, we conclude that the PAMFPAD of $\mathrm{CH}_{4}$ indeed images the molecular geometry and that the nuclear motion has a very minor effect on the shape of the vibrationally unresolved MFPADs.

Figure 3 shows the PAMFPADs for $K$-shell ionization of $\mathrm{CO}, \mathrm{N}_{2}, \mathrm{C}_{2} \mathrm{H}_{2}, \mathrm{NH}_{3}, \mathrm{CH}_{4}, \mathrm{CF}_{4}, \mathrm{BF}_{3}$, and $\mathrm{SF}_{6}$ at some selected photoelectron energies [29]. As can be seen, the

\footnotetext{
${ }^{1}$ Feshbach resonances associated with doubly excited states may also appear close to the ionization threshold; however, they cannot be described by the present static-exchange DFT method.
}
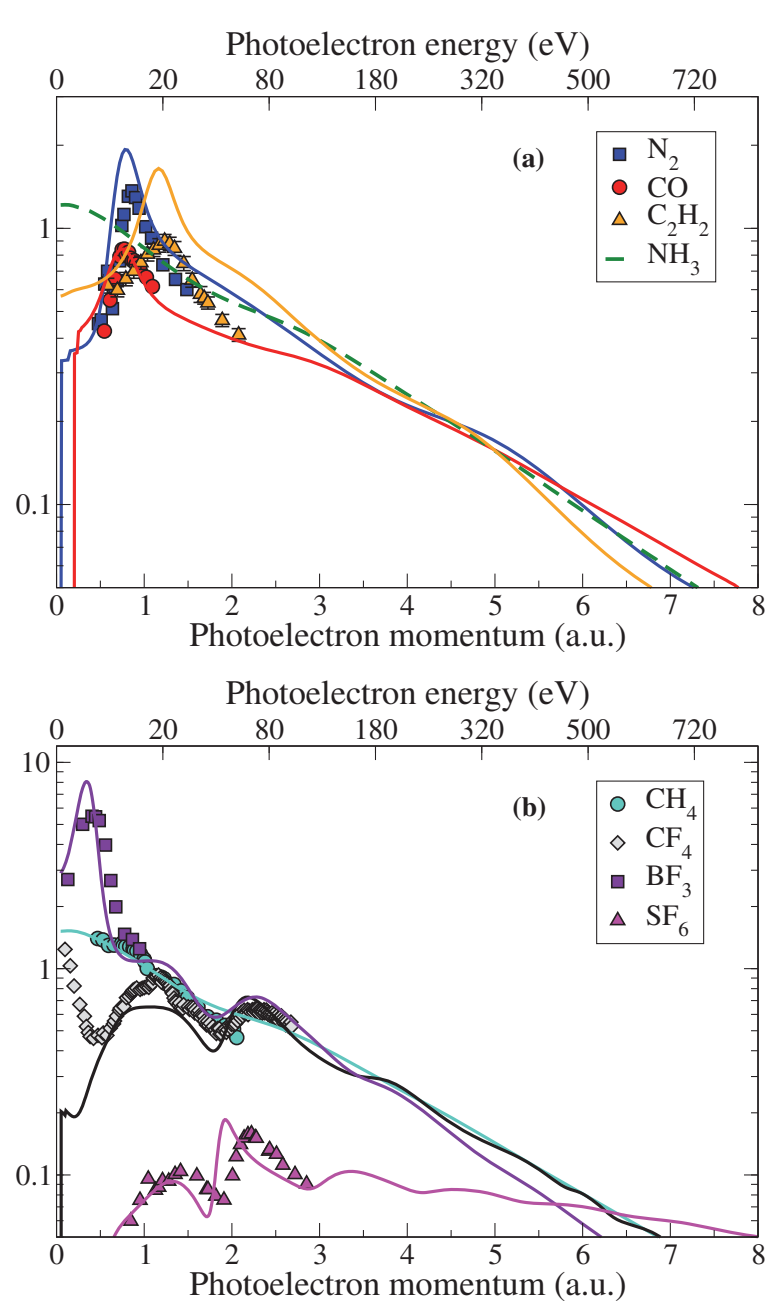

FIG. 1. (Color online) Cross sections as functions of photoelectron energy. (a) Lines: present calculations for $\mathrm{N}_{2}$ (blue [dark gray]), $\mathrm{CO}$ (red [gray]), $\mathrm{C}_{2} \mathrm{H}_{2}$ (orange [light gray]), and $\mathrm{NH}_{3}$ (dashed line). Symbols: experimental results from Refs. [22] ( $\mathrm{N}_{2}$, squares), [23] ( $\mathrm{CO}$, renormalized data, circles), and [24] $\left(\mathrm{C}_{2} \mathrm{H}_{2}\right.$, triangles). (b) Lines: present calculations for $\mathrm{CH}_{4}$ (turquoise [light gray]), $\mathrm{CF}_{4}$ (gray), $\mathrm{BF}_{3}$ (violet [dark gray]), and $\mathrm{SF}_{6}$ (pink [pale gray]). Symbols: experimental results from Refs. [25] $\left(\mathrm{CH}_{4}\right.$, circles), [26] ( $\mathrm{CF}_{4}$, diamonds), [27] $\left(\mathrm{BF}_{3}\right.$, squares $)$, and [28] ( $\mathrm{SF}_{6}$, triangles). The photoelectron energy is given by [ $h v-\mathrm{IP}]$, where IP is the energy required to remove an electron from the $K$ shell of the molecule by leaving the cation in the ground vibrational state.

PAMFPADs are far from being isotropic even at photoelectron energies as high as $500 \mathrm{eV}$. In general, they largely differ from molecule to molecule and behave very differently as a function of photoelectron energy. This is in spite of the fact that some molecules, like $\mathrm{CH}_{4}$ and $\mathrm{CF}_{4}$, have a similar structure and belong to the same symmetry group $\left(\mathrm{T}_{d}\right)$. At very low photoelectron energy, say below $10 \mathrm{eV}$, the PAMFPADs of $\mathrm{NH}_{3}$ and $\mathrm{CH}_{4}$ (with $\mathrm{C}_{3 v}$ and $\mathrm{T}_{d}$ point group symmetries, respectively) image the molecular geometry. These two distributions become nearly isotropic at around $30-40 \mathrm{eV}$, then reflect again the molecular geometry up to $100-200 \mathrm{eV}$, and then become again nearly isotropic (even more than at 30-40 eV). The vibrationally resolved PAMFPADs, among which more than $90 \%$ correspond to leaving $\mathrm{CH}_{4}^{+}$and $\mathrm{NH}_{3}^{+}$ 


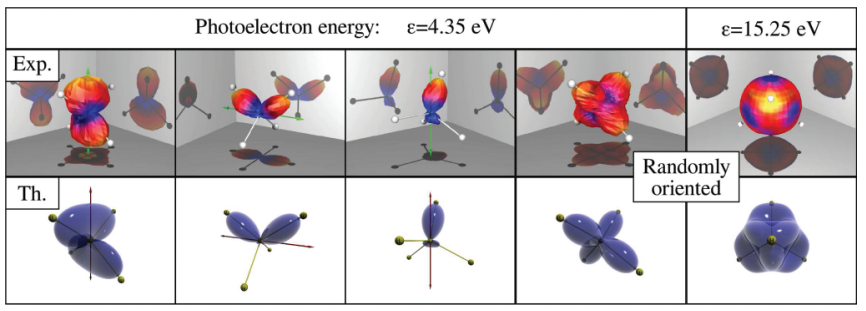

FIG. 2. (Color online) MFPADs and MFPADs averaged over the polarization direction for $K$-shell ionization of $\mathrm{CH}_{4}$ at two photoelectron energies, 4.35 and $15.25 \mathrm{eV}$. Top row: experimental data from Refs. $[14,15]$. Bottom row: present calculations. The arrow represents the polarization direction.

in the $v=0$ ground vibrational state, behave in the same way. For the linear molecules $\mathrm{N}_{2}$ and $\mathrm{C}_{2} \mathrm{H}_{2}\left(\mathrm{D}_{\infty h}\right.$ symmetry) and $\mathrm{CO}\left(\mathrm{C}_{\infty v}\right.$ symmetry), the PAMFPADs never image the molecular geometry, even at very low photoelectron energy. As discussed in Refs. [18,30], for $\mathrm{N}_{2}$ and, consequently, for $\mathrm{C}_{2} \mathrm{H}_{2}$, confinement effects, due to the transient trapping of the photoelectron when the latter escapes along the bond and its wavelength matches twice the N-N or C-C internuclear distances, respectively, are expected to occur. This has been predicted and observed in $K$-shell photoionization of $\mathrm{N}_{2}, \mathrm{CO}$, and $\mathrm{H}_{2}[30,31]$ and it is the reason why the PAMFPAD of $\mathrm{C}_{2} \mathrm{H}_{2}$ exhibits a sort of diabolo shape at around $200 \mathrm{eV}$ : It simply reflects the fact that the photoelectron does not escape preferentially along the internuclear axis. At these energies, the additional perpendicular lobes are simply due to double-slit interferences as those already predicted and observed in $K$-shell ionization of $\mathrm{N}_{2}[5,18,30]$ and $\mathrm{H}_{2}[4,31,32]$.

$\mathrm{CH}_{4}$ and $\mathrm{CF}_{4}$ belong to the same symmetry group, $T_{d}$; however, their PAMFPADs are quite different. Close to the ionization threshold, for $\epsilon<12 \mathrm{eV}$, the $\mathrm{CF}_{4}$ PAMFPAD changes dramatically with photoelectron energy. This is the region where the cross section exhibits peaks associated with shape resonances (see Fig. 1). In this region, not only does the PAMFPAD not image the molecular geometry but also the main lobes lie preferentially in between the C-F bonds. For $\epsilon>150 \mathrm{eV}$, the PAMFPADs are mainly composed of two parts: The first one, consisting of four lobes along the C-F bonds, barely changes with $\epsilon$; the second one, which lies around the central $\mathrm{C}$ atom, oscillates with $\epsilon$ through successive implosions and explosions, increasing the number of lobes after each implosion. The PAMFPADs of the planar molecule $\mathrm{BF}_{3}$ (point group symmetry $D_{3 h}$ ) and the octahedral molecule $\mathrm{SF}_{6}\left(O_{h}\right)$ follow a similar pattern: At low energies, the main lobes lie in between the molecular bonds, even more clearly than in $\mathrm{CF}_{4}$; at high energies, the main lobes follow the bonds irrespective of $\epsilon$ and the breathing of the central structure is systematically accompanied by the appearance of new lobes as $\epsilon$ increases.

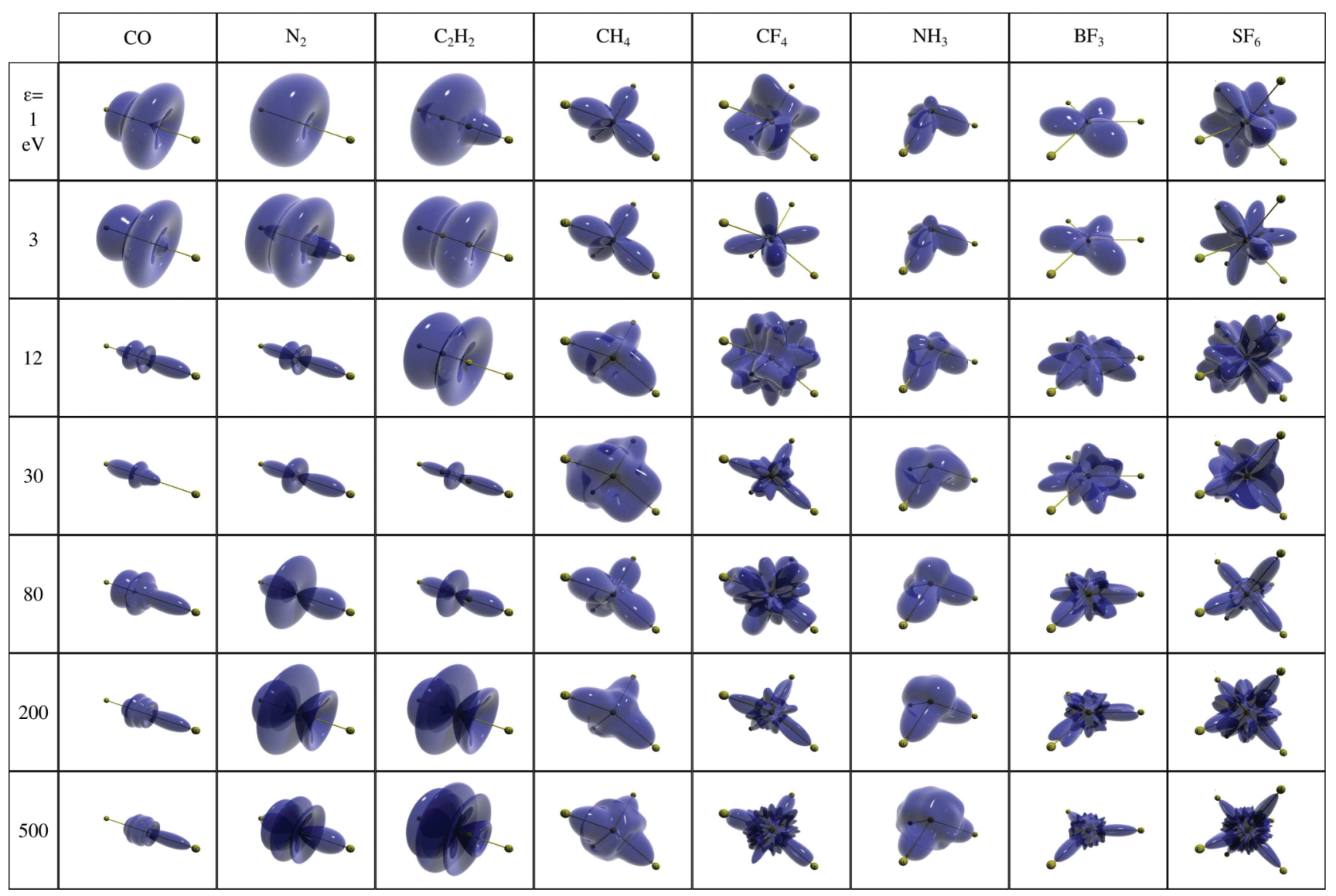

FIG. 3. (Color online) MFPADS averaged over polarization directions for $K$-shell ionization of $\mathrm{CO}$ [C $\left.\left(1 \mathrm{~s}^{-1}\right)\right], \mathrm{N}_{2}, \mathrm{C}_{2} \mathrm{H}_{2}, \mathrm{NH}_{3}, \mathrm{CH}_{4}, \mathrm{CF}_{4}$ [C $\left.\left(1 \mathrm{~s}^{-1}\right)\right], \mathrm{BF}_{3}\left[\mathrm{~B}\left(1 \mathrm{~s}^{-1}\right)\right]$, and $\mathrm{SF}_{6}\left[\mathrm{~S}\left(1 \mathrm{~s}^{-1}\right)\right]$, in the range of photoelectron energies $0-500 \mathrm{eV}$. For better visualization, all MFPADs have been renormalized to the same maximum value. 


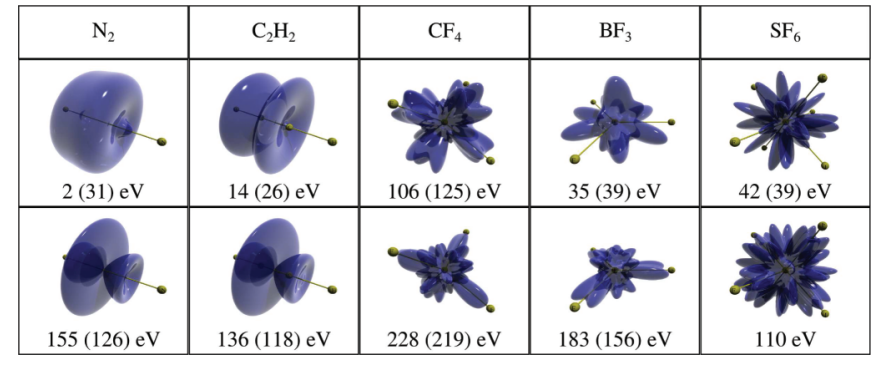

FIG. 4. (Color online) PAMFPADs exhibiting the smallest lobes along the bonds relative to the central lobes. PAMFPADs are renormalized as in Fig. 3. The corresponding photoelectron energies are compared to the eigenenergies (within parentheses) of an electron contained in a one-dimensional $\left(\mathrm{N}_{2}\right.$ and $\left.\mathrm{C}_{2} \mathrm{H}_{2}\right)$, triangular $\left(\mathrm{BF}_{3}\right)$, tetrahedral $\left(\mathrm{CF}_{4}\right)$, and octahedral $\left(\mathrm{SF}_{6}\right)$ potential box. The latter three have been obtained by using the experimental bond distances in the formulas given, respectively, in Refs. [33,34], and [34,35] (see text).

A possible explanation for this behavior is the following: Fluorine is a very electronegative element and, therefore, has an excess of negative charge. Thus, slow electrons escaping from the central atom are repelled by the negatively charged $\mathrm{F}$ atoms and escape preferentially in between the bonds. In contrast, at high $\epsilon$, the electron can efficiently penetrate the electron cloud and feel the strong nuclear attraction, so that it escapes preferentially along the bonds and is efficiently diffracted by the F nuclei. Although the mean potential felt by the photoelectron is attractive everywhere, the photoelectron can still be transiently trapped in between the central and the peripheral atoms when its energy approximately matches that of a virtual single-electron state of the molecule. The effect is similar to that observed in the resonant scattering of a particle by an attractive potential box of finite depth at positive impact energies and does not require the existence of potential barriers. It is also similar to the confinement effect described in Refs. [30,31] and above for diatomic molecules, and for $\mathrm{C}_{2} \mathrm{H}_{2}$ (in the latter case, confinement only occurs in between the two $\mathrm{C}$ atoms). To check the validity of this interpretation in the case of fluorine substituted molecules, we have used the formulas reported in Refs. [33-35] to calculate the eigenvalues of the triangular, tetrahedral, and octahedral potential boxes that are built by assuming that the distance between the geometric center of the box and the vertices is equal to the bond length. ${ }^{2}$ In Fig. 4, we compare these eigenvalues with the photoelectron energies at which the PAMFPADs exhibit the largest relative contributions in the central part of the molecule. For completeness, we have also added the results for $\mathrm{N}_{2}$ and $\mathrm{C}_{2} \mathrm{H}_{2}$, as well as the PAMFPADs at the corresponding photoelectron energies. As can be seen, there is qualitative agreement between photoelectron energies and box eigenvalues.

\footnotetext{
${ }^{2}$ For the triangular box, we have used the known exact formula from Ref. [33]. For the tetrahedral and octahedral boxes, as only variational approximations to the ground-state energy are known [34], the calculated eigenvalues are only approximate; the second lowest eigenenergy of the tetrahedral box is not known, so we have used that of a $K$-tetrahedron [35] rescaled so that the ground-state energies of both tetrahedra are identical.
}

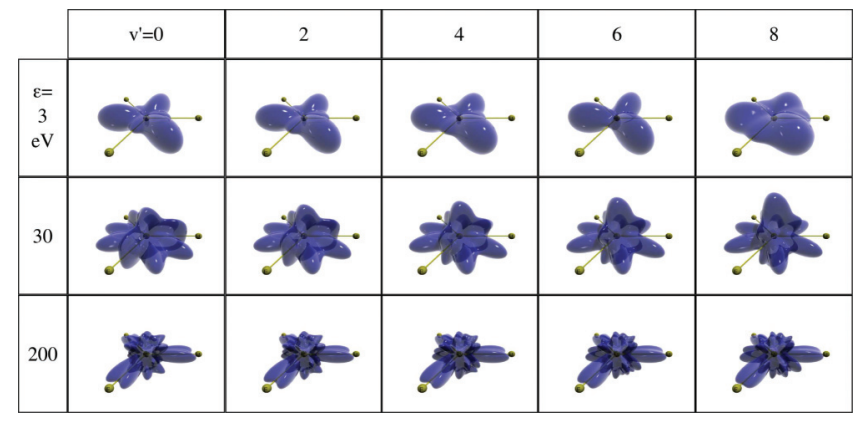

FIG. 5. (Color online) Vibrationally resolved PAMFPADs resulting from $K$-shell ionization of $\mathrm{BF}_{3}$ for three photoelectron energies and for the final $v=0-8$ vibrational states of $\mathrm{BF}_{3}^{+}$. PAMFPADs are renormalized as in Fig. 3.

We turn now our attention to the vibrationally resolved MFPADs. By far, for all molecules investigated here, the dominant vibrational progression corresponds to the symmetric stretching mode. The largest progression is obtained for $\mathrm{BF}_{3}$. $K$-shell photoionization of this molecule leads to a significant population of $\mathrm{BF}_{3}^{+}$cations in vibrational states from $v=0$ to $v=7$ [36]; among them, the largest probability corresponds to $v=2-3$. For all the other polyatomic molecules, the dominant ionization channel is $v=0$, and the vibrational progression is limited to two or at most three vibrational levels [37,38]. Thus $\mathrm{BF}_{3}$ is the ideal system to investigate changes in the MFPADs due to the nuclear motion. Figure 5 shows the vibrationally resolved PAMFPADs for this molecule at three selected values of the photoelectron energies given in Fig. 3. By comparing the results of Fig. 5 with the movies for the unresolved PAMFPADs given in the Supplemental Material [29], one can see that PAMFPADs for high $v$ look like unresolved PAMFPADs at slightly higher photoelectron energy. This can be explained as follows. Although for all vibrationally resolved PAMFPADs the energy carried by the electron is the same, the average B-F distance that the electron sees increases as $v$ increases due to anharmonicity. Therefore, electrons that leave $\mathrm{BF}_{3}^{+}$in a high $v$ will see a larger potential box, so that the corresponding energy eigenvalues will be shifted down with respect to the original box and the escaping electron will behave in practice as having a larger relative energy.

\section{CONCLUSION}

In summary, direct imaging of molecular geometry by means of $K$-shell PAMFPADs is only observed for simple hydrides at very low and selected high kinetic energies. For all other molecules, namely diatomics (or quasidiatomics) and molecules containing a central atom surrounded by atoms significantly heavier than $\mathrm{H}$, the PAMFPADs do not reflect the system's geometry. Still, the PAMFPADs for the latter molecules reflect partial accumulation of the photoelectron density in the region surrounded by the peripheral atoms, which encodes information about the three-dimensional arrangement of the system. In general, the PAMFPADs remain anisotropic even at photoelectron energies as high as $500 \mathrm{eV}$. 


\section{ACKNOWLEDGMENTS}

We thank Mare Nostrum BSC, Cineca and CCC-UAM for allocation of computer time. This work was supported by the Advanced Grant of the European Research Council
XCHEM 290853, the MICINN Projects No. FIS2010-15127 and No. CSD 2007-00010 (Spain), the ERA-Chemistry Project PIM2010EEC-00751, the European Grant MC-ITN CORINF, and the European COST Actions CM0702 and CM1204.
[1] A. H. Zewail, Science 328, 187 (2010).

[2] H. N. Chapman et al., Nature (London) 470, 73 (2011).

[3] C. I. Blaga, J. Xu, A. D. DiChiara, E. Sistrunk, K. Zhang, P. Agostini, T. A. Miller, L. F. DiMauro, and C. D. Lin, Nature (London) 483, 194 (2012).

[4] S. E. Canton, E. Plésiat, J. D. Bozek, B. S. Rude, P. Decleva, and F. Martín, Proc. Natl. Acad. Sci. USA 108, 7302 (2011).

[5] X.-J. Liu, N. A. Cherepkov, S. K. Semenov, V. Kimberg, F. Gel'mukhanov, G. Prümper, T. Lischke, T. Tanaka, M. Hoshino, H. Tanaka et al., J. Phys. B: At. Mol. Opt. Phys. 39, 4801 (2006).

[6] L. Argenti, T. D. Thomas, E. Plésiat, X.-J. Liu, C. Miron, T. Lischke, G. Prümper, K. Sakai, T. Ouchi, R. Püttner et al., New J. Phys. 14, 033012 (2012).

[7] E. Plésiat, L. Argenti, E. Kukk, C. Miron, K. Ueda, P. Decleva, and F. Martín, Phys. Rev. A 85, 023409 (2012).

[8] H. Fukuzawa, X.-J. Liu, T. Teranishi, K. Sakai, G. Prümper, K. Ueda, Y. Morishita, N. Saito, M. Stener, and P. Decleva, Chem. Phys. Lett. 451, 182 (2008).

[9] J. Adachi, M. Kazama, T. Teramoto, N. Miyauchi, T. Mizuno, M. Yamazaki, T. Fujikawa, and A. Yagishita, J. Phys. B 45, 194007 (2012).

[10] J. J. Larsen, K. Hald, N. Bjerre, H. Stapelfeldt, and T. Seideman, Phys. Rev. Lett. 85, 2470 (2000).

[11] L. Holmegaard et al., Nat. Phys. 6, 428 (2010).

[12] R. Dörner, V. Mergel, O. Jagutzki, L. Spielberger, J. Ullrich, R. Moshammer, and H. Schmidt-Böcking, Phys. Rep. 330, 95 (2000).

[13] M. Kazama, H. Shinotsuka, T. Fujikawa, M. Stener, P. Decleva, J. Adachi, T. Mizuno, and A. Yagishita, J. Electron Spectroc. Relat. Phenom. 185, 535 (2012).

[14] J. B. Williams, C. S. Trevisan, M. S. Schöffler, T. Jahnke, I. Bocharova, H. Kim, B. Ulrich, R. Wallauer, F. Sturm, T. N. Rescigno et al., Phys. Rev. Lett. 108, 233002 (2012).

[15] J. B. Williams, C. S. Trevisan, M. S. Schöffler, T. Jahnke, I. Bocharova, H. Kim, B. Ulrich, R. Wallauer, F. Sturm, T. N. Rescigno et al., J. Phys. B: At. Mol. Opt. Phys. 45, 194003 (2012).

[16] C. S. Trevisan, C. W. McCurdy, and T. N. Rescigno, J. Phys. B: At. Mol. Opt. Phys. 45, 194002 (2012).

[17] J.-I. Adachi, K. Hosaka, S. Furuya, K. Soejima, M. Takahashi, A. Yagishita, S. K. Semenov, and N. A. Cherepkov, Phys. Rev. Lett. 91, 163001 (2003).

[18] E. Plésiat, P. Decleva, and F. Martín, Phys. Chem. Chem. Phys. 14, 10853 (2012).
[19] M. Stener, G. De Alti, and P. Decleva, Theor. Chem. Acc. 101, 247 (1999).

[20] M. Stener and P. Decleva, J. Chem. Phys. 112, 10871 (2000).

[21] H. Bachau, E. Cormier, P. Decleva, J. E. Hansen, and F. Martín, Rep. Prog. Phys. 64, 1815 (2001).

[22] S. K. Semenov, N. A. Cherepkov, M. Matsumoto, K. Fujiwara, K. Ueda, E. Kukk, F. Tahara, T. Sunami, H. Yoshida, T. Tanaka et al., J. Phys. B: At. Mol. Opt. Phys. 39, 375 (2006).

[23] D. A. Mistrov, A. De Fanis, M. Kitajima, M. Hoshino, H. Shindo, T. Tanaka, Y. Tamenori, H. Tanaka, A. A. Pavlychev, and K. Ueda, Phys. Rev. A 68, 022508 (2003).

[24] M. Hoshino, K. Nakagawa, C. Makochekanwa, T. Tanaka, N. Kuze, M. Matsumoto, K. Fujiwara, A. De Fanis, Y. Tamenori, M. Kitajima et al., Chem. Phys. Lett. 421, 256 (2006).

[25] H. M. Köppe, B. S. Itchkawitz, A. L. D. Kilcoyne, J. Feldhaus, B. Kempgens, A. Kivimäki, M. Neeb, and A. M. Bradshaw, Phys. Rev. A 53, 4120 (1996).

[26] A. P. Hitchcock and D. C. Mancini, J. Electron Spectrosc. Relat. Phenom. 67, vii (1994).

[27] Y. Shimizu, K. Ueda, H. Chiba, and K. Ohmori, J. Chem. Phys. 107, 2415 (1997).

[28] T. A. Ferrett, D. W. Lindle, P. A. Heimann, H. G. Kerkhoff, U. E. Becker, and D. A. Shirley, Phys. Rev. A 34, 1916 (1986).

[29] See Supplemental Material at http://link.aps.org/supplemental/ 10.1103/PhysRevA.88.063409 for movies showing the detailed variation of the PAMFPADs with photoelectron energy.

[30] E. Plésiat, P. Decleva, and F. Martín, J. Phys. B: At. Mol. Opt. Phys. 45, 194008 (2012).

[31] J. Fernández, O. Fojón, A. Palacios, and F. Martín, Phys. Rev. Lett. 98, 043005 (2007).

[32] J. Fernández, O. Fojón, and F. Martín, Phys. Rev. A 79, 023420 (2009).

[33] W.-K. Li and S. M. Blinder, J. Math. Phys. 26, 2784 (1985).

[34] W.-K. Li and S. M. Blinder, Chem. Phys. Lett. 496, 339 (2010).

[35] H. R. Krishnamurthy, H. S. Mani, and H. C. Verma, J. Phys. A 15, 2131 (1982).

[36] T. D. Thomas, R. Püttner, H. Fukuzawa, G. Prümper, K. Ueda, E. Kukk, R. Sankari, J. Harries, Y. Tamenori, T. Tanaka et al., J. Chem. Phys. 127, 244309 (2007).

[37] E. Kukk, K. Ueda, U. Hergenhahn, X.-J. Liu, G. Prümper, H. Yoshida, Y. Tamenori, C. Makochekanwa, T. Tanaka, M. Kitajima et al., Phys. Rev. Lett. 95, 133001 (2005).

[38] T. D. Thomas, E. Kukk, R. Sankari, H. Fukuzawa, G. Prümper, K. Ueda, R. Püttner, J. R. Harries, Y. Tamenori, T. Tanaka et al., J. Chem. Phys. 128, 144311 (2008). 